\title{
DAMAGE CORRELATIONS IN SEMICONDUCTORS EXPOSED TO GAMMA, ELECTRON AND PROTON RADIATIONS
}

\author{
Geoffrey P. Summers \\ Naval Research Laboratory, Washington, DC 20375 and \\ Department of Physics \\ University of Maryland Baltimore County, Baltimore, MD 21228 \\ Edward A. Burke, Philip Shapiro and Scott R. Messenger \\ SFA, Inc., Landover, MD 20785 \\ Robert J. Walters \\ Naval Research Laboratory, Washington, DC 20375
}

\begin{abstract}
The use of nonionizing energy loss (NIEL) in predicting the effect of gamma, electron and proton irradiations on Si, GaAs and InP devices is discussed. The NIEL for electrons and protons has been calculated from the displacement threshold to $200 \mathrm{MeV}$. Convoluting the electron NIEL with the "slowed down" Compton secondary electron spectrum gives an effective NIEL for $\mathrm{Co}^{60}$ gammas, enabling gamma-induced displacement damage to be correlated with particle results. The fluences of $1 \mathrm{MeV}$ electrons equivalent to irradiation with $1 \mathrm{Mrad}(\mathrm{Si})$ for $\mathrm{Si}, \mathrm{GaAs}$ and $\mathrm{InP}$ are given. Analytic proton NIEL calculations and results derived from the Monte Carlo code TRIM agree exactly so long as straggling is not significant. The NIEL calculations are compared with experimental proton and electron damage coefficients using solar cells as examples. A linear relationship is found between the NIEL and proton damage coefficients for $\mathrm{Si}, \mathrm{GaAs}$ and InP devices. For electrons, there appears to be a linear dependence for $\mathrm{n}-\mathrm{Si}$ and $\mathrm{n}-\mathrm{GaAs}$, but for $\mathrm{p}-\mathrm{Si}$ there is a quadratic relationship which decreases the damage coefficient at $1 \mathrm{MeV}$ by a factor of $\sim 10$ below the value for $\mathrm{n}-\mathrm{Si}$. The present results greatly extend the range of environments for which damage calculations based on NIEL can be applied. The NIEL results are presented in tabular form for ease of calculation.
\end{abstract}

\section{INTRODUCTION}

In a previous paper [1], it was shown that the displacement damage effect of high energy ( $>2 \mathrm{MeV}$ ) protons, deuterons and helium ions on the gain of $\mathrm{Si}$ bipolar transistors could be correlated on the basis of analytic calculations of nonionizing energy loss (NIEL). NIEL is a calculation of the rate of energy loss due to atomic displacements as an incident particle traverses a material, and the product of the NIEL and the particle fluence gives the displacement damage energy along the track. The finding of a linear relationship between the NIEL and experimental damage coefficients suggested a method of calculating the radiation response of $\mathrm{Si}$ devices in a complex proton space environment, using only one or two ground tests and the energy dependence of the proton NIEL. In subsequent papers a similar approach was discussed for $\mathrm{GaAs}[2], \mathrm{Ge}[3]$ and InP[4] devices, and also for high temperature superconductors[5].

A device in an actual space environment is irradiated with a spectrum of proton and electron energies, so that even if shielding is employed, the particles traversing a particular point in the device will possess a range of energies, from a few $\mathrm{eV}$ up to $\sim 100 \mathrm{MeV}$. This issue was not addressed in the early papers, in which it was assumed that the incident particles did not lose significant energy in traversing the active region of the device and that the damage was therefore produced by a monoenergetic beam. In order to calculate the damage energy produced by a spectrum of particles, it is necessary to know both the NIEL for energies from the threshold for displacement up to high energy and the particle spectrum at a point in the device, obtained using a suitable transport code, for example. In this paper, NIEL calculations are given in tabular form for $\mathrm{Si}, \mathrm{GaAs}$ and InP from the threshold up to $200 \mathrm{MeV}$ so that other users can make damage estimates for their own particular environments.

Protons in a particular space environment are expected to produce most of the damage and the effect of any electrons present can often be neglected. Fortunately, the dependence of proton damage coefficients on NIEL has been found to be linear in all cases reported, so the calculation is straightforward. However, the situation for electron environments is more complicated, as will be discussed in Section IIIB. The calculation of electron NIEL is examined in detail because of its importance in analyzing this more complicated behavior.

The relationship between proton and electron damage is especially important in the solar photovoltaics community, where it is usual to compare different technologies by their response to $1 \mathrm{MeV}$ electrons, even when the expected environment consists primarily of protons[6]. In this approach, the effect of the proton environment is reduced to a $10 \mathrm{MeV}$ proton equivalent fluence (i.e., the fluence of $10 \mathrm{MeV}$ protons that produces the same damage effect as the actual spectrum), which in turn is converted to an equivalent $1 \mathrm{MeV}$ electron fluence. This fluence is added to the result of a similar calculation for the electron spectrum. It would simplify the calculation if the conversion from a $10 \mathrm{MeV}$ proton fluence to an equivalent $1 \mathrm{MeV}$ electron fluence could be made analytically using only the respective NIELs, but this appears to be possible only in selected cases. 
Finally, as particle accelerators become less accessible and their use more costly, it is advantageous to investigate the use of alternative radiation sources such as $\mathrm{Co}^{60}$ for making displacement damage measurements. A $\mathrm{Co}^{60}$ source also has the advantage that the irradiation conditions, such as the damage rate, can be varied easily to simulate conditions not normally accessible at accelerators, making an experimental study of the interrelation of damage rate, annealing and temperature effects relatively straightforward.

\section{NONIONIZING ENERGY LOSS}

Calculation of proton NIEL for energies $>1 \mathrm{MeV}$ has been discussed in detail previously[7], especially the contribution to the total NIEL from nuclear elastic and inelastic interactions. The new results given here extend down to the displacement threshold energy, where the interactions are entirely Rutherford. For energies $<50 \mathrm{keV}$ the nonrelativistic cross sections given by Seitz and Koehler[8] were used in place of the relativistic form employed at higher energies.

At low proton and electron energies, the NIEL is sensitive to the values of the threshold energy used. The values used here were 12.9 and $21 \mathrm{eV}$ for $\mathrm{Si}$ [9], $10 \mathrm{eV}$ for $\mathrm{Ga}$ and for As [10], 6.7 eV for In and $8.7 \mathrm{eV}$ for $\mathrm{P}$ [11].

\section{IIA. Electron NIEL} integral:

Calculation of NIEL reduces to an evaluation of the

$$
\begin{aligned}
& N I E L=(N / A) \int_{L[T(\theta)] T(\Theta) d \sigma(\Theta) / d \Omega d \Omega}^{180} \\
& \theta \mathrm{min}
\end{aligned}
$$

where $\mathbf{N}$ is Avogadro's number, $\mathbf{A}$ is the atomic mass, and $T(\Theta)$ is the energy transferred to the target nucleus by an electron scattered through an angle $\theta$ in the center of mass system. Maximum energy transfer occurs for $\Theta=180^{\circ}$. $\mathrm{d} \sigma(\Theta) / \mathrm{d} \Omega$ is the differential cross section for elastic scattering of electrons into a solid angle increment $d \Omega$ and $L[T(\theta)]$ is the Lindhard partition factor [12], which gives the fraction of transferred energy that is nonionizing. The lower limit on the integral, $\boldsymbol{\theta}_{\min }$, is the scattering angle for which the recoil energy equals the threshold for displacement.

For ease of computation, it is necessary to make approximations to both the Mott differential cross sections[13] and to the Lindhard partition function[12]. For the differential cross sections, the McKinley-Feshbach[14] approximation can be used for low $\mathrm{Z}$ materials including $\mathrm{Si}$, but for higher $\mathrm{Z}$ (> 24) atoms such as In, the Curr[15] form is more accurate. Curr only gives parameters for specific values of the scattering angle down to a minimum of $30^{\circ}$, however, and for smaller angles, the parameters given by Dogget and Spencer[16] were employed.

An approximate form for the Lindhard partition function which is incorporated in the damage code TRIM, is given by Ziegler et al.[17]. This approximation is similar to one given by Doran[18]. At low recoil energies, both approximations overestimate the losses to ionization, and Sattler[19] gives an alternative form based on Lindhard's original work, which is valid for energies $E(k e V)<<A Z / 2$. For computational purposes in the work described here, the Sattler form was used for recoil energies less than the energy where the two approaches give the same result. This recoil energy for $\mathrm{Si}$ is $\sim 28.4 \mathrm{keV}$. At higher energies the Doran approximation was employed.

Figure 1 shows the proton and electron NIEL for GaAs from the threshold energies up to $100 \mathrm{MeV}$. Tabulated values up to $200 \mathrm{MeV}$ are given in the Appendix, where the results for Si and InP are also presented. Separate calculations were performed using both $21 \mathrm{eV}$ and $12.9 \mathrm{eV}$ for the threshold energy for $\mathrm{Si}$. A value of $2 \mathrm{l} \mathrm{eV}$ fits much of the data better as shown by Watkins and Corbett[9], but Bauerlein [11] and others have quoted the lower value of $12.9 \mathrm{eV}$.

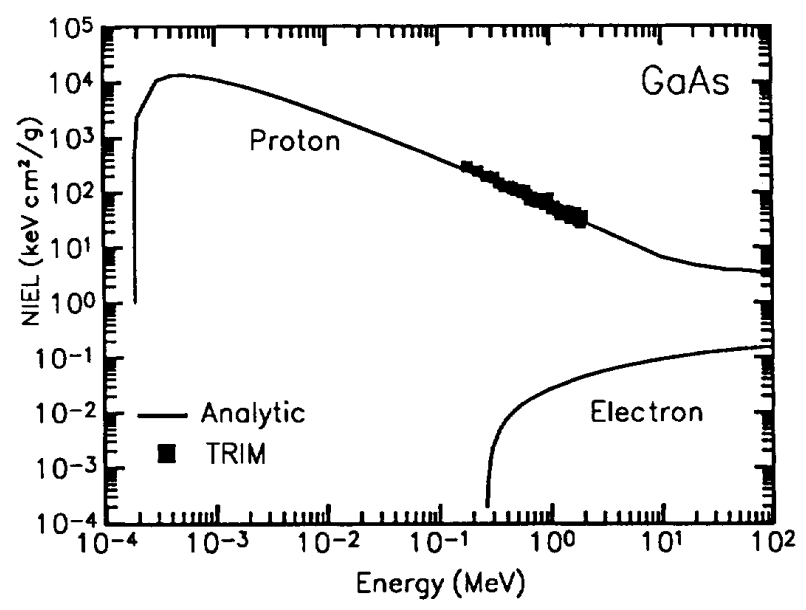

Figure 1 NIEL for protons and electrons in GaAs. The squares show results based on TRIM for incident $2 \mathrm{MeV}$ protons. No fitting parameters have been used.

\section{IIB. Comparison of Analytic Calculations with TRIM}

The Monte Carlo code TRIM[20] is widely used to obtain information about vacancy production rates. With some manipulation of the output files, TRIM can also be employed to calculate NIEL for comparison with the analytic calculations discussed above. The TRIM output gives the vacancy production rate as a function of position as the incident proton slows down in the target material. Combining these data with the total energy loss data, the vacancy production rate as a function of proton energy can be found. The vacancy concentration can be converted to damage energy using the modified Kinchin-Pease approximation, which then yields the NIEL as a function of proton energy. The data points in figure 1 are calculated in this way for incident $2 \mathrm{MeV}$ protons in GaAs, down to an energy of $0.2 \mathrm{MeV}$ The agreement with the analytic calculations can be seen to be 
excellent with no fitting parameters applied.

TRIM performs a Monte Carlo calculation and straggling occurs as the proton loses energy. Straggling has the effect of reducing the NIEL below the analytic value, at the end of the proton track. This effect is not shown in figure 1 where the TRIM results are terminated at $0.2 \mathrm{MeV}$. However, if the calculation is repeated using a lower energy incident proton, the agreement with the analytic result is maintained to a correspondingly lower energy.

Because only Rutherford interactions are used in TRIM, the NIEL for proton energies $>-12 \mathrm{MeV}$ in GaAs cannot be calculated in this way. However, the analytic calculations can be extended up to much higher energies as shown in figure 1 . The TRIM and the analytic calculations therefore complement each other in a useful way.

\section{IIC. Co60 Gamma-induced Displacement Damage}

$\mathrm{Co}^{60}$ gammas are widely used to study ionizationinduced damage in devices incorporating dielectrics, such as MOSFETs. However, so long as their energy is above the threshold, the secondary electrons produced by incident gammas are also able to displaceme atoms. $\mathrm{Co}^{60}$ sources are convenient for such studies only if the displacement damage produced can be correlated with that induced by other particles. In this section, a determination of the average effective NIEL for $\mathrm{Co}^{60}$ gammas will be described, which makes this correlation possible.

The basic assumption in the calculation is that the displacements are caused by secondary electrons produced by $\mathrm{Co}^{60}$ gammas mainly in the material surrounding the device under study. This condition will be met so long as no high $\mathrm{Z}$ (> 29) materials are nearby, as is often the case when aluminum, concrete, air or water surrounds the device. Compton electrons dominate the spectrum and the broken line in figure 2 shows the primary Compton electron flux produced in $\mathrm{Al}$ by $\mathrm{Co}^{60}$ gammas. However, the electrons incident on a small volume in a sample have already traversed some material and the electron flux consists of the so called "slowed down" spectrum, shown as the continuous line in figure 2 . The Compton cross section is relatively insensitive to the atomic number, unlike the photoelectric cross section, so the calculation made here for $\mathrm{Al}$ would change little for other materials. Figure 2 shows a normalized curve for the secondary electron flux. The ratio of the electron flux to the gamma photon flux in $\mathrm{Al}$ is 0.0162 .

When the "slowed down" spectrum is combined with the energy dependence of the electron NIEL given in the Appendix, the average NIEL value for gamma-induced secondary electrons can be obtained. These NIEL values are shown for $\mathrm{Si}, \mathrm{GaAs}$ and InP in Table 1.

The average gamma-induced NIEL values shown in Table 1 enable the fluences of $1 \mathrm{MeV}$ electrons equivalent to irradiating $\mathrm{Si}, \mathrm{GaAs}$, and InP with $1 \mathrm{Mrad}(\mathrm{Si})$ to be determined. In making this calculation, the relationship between NIEL and damage coefficient as a function of electron energy must be known. As will be apparent later, this relationship may not be linear. For p-type $\mathrm{Si}$, it is quadratic, which leads to a different I MeV equivalent electron fluence for $\mathrm{n}$ - and $\mathrm{p}$-type $\mathrm{Si}$, as shown in Table 1.

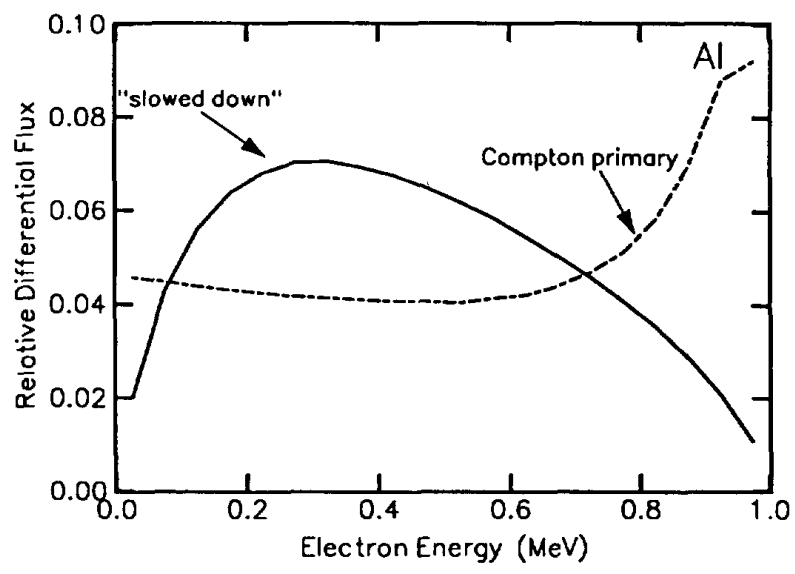

Figure 2 Differential flux of Compton secondary electrons in $\mathrm{Al}$ for both the primary and for the "slowed down" spectra.

TABLE 1

$\mathrm{Co}^{60}$ GAMMA-INDUCED DISPLACEMENT DAMAGE 1

$\begin{array}{lll}\text { Average } \gamma \text {-induced } & 1 \mathrm{MeV} \text { Electron } & \text { Equivalent } 1 \mathrm{MeV} \\ \text { Electron NIEL } & \text { NIEL } & \text { Electron Fluence } \\ \left(\mathrm{eV} . \mathrm{cm}^{2} / \mathrm{g}\right) & \left(\mathrm{eV} . \mathrm{cm}^{2} / \mathrm{g}\right) & \begin{array}{l}\text { for } 1 \mathrm{Mrad}(\mathrm{Si}) \\ \left(\mathrm{cm}^{-2}\right)\end{array}\end{array}$

$\begin{array}{lccr}\text { n-Si } & 13.08 & 31.42 & 1.25 \times 10^{13} \\ \text { p-Si } & & 31.42 & 8.04 \times 10^{12} \\ \text { GaAs } & 9.25 & 26.5 & 1.08 \times 10^{13} \\ \text { InP } \quad 14.19 & 33.5 & 1.30 \times 10^{13} \\ & & \\ \text { I Threshold energies: } \mathrm{Si}=21 \mathrm{eV}, \mathrm{Ga} \text { and } \mathrm{As}=10 \mathrm{eV}, \\ \text { In }=6.7 \mathrm{eV} \text { and } \mathrm{P}=8.7 \mathrm{eV} .\end{array}$

Data in the literature for $\mathrm{Co}^{60}$ gamma and $1 \mathrm{MeV}$ electron damage coefficients are usually presented without information about details of the gamma exposure, so that quantitative comparison with the results shown in Table 1 is difficult. However, the reported data for Si[6], GaAs[21] and InP[22] are within the uncertainty associated with such effects.

\section{COMPARISON OF NIEL WITH DAMAGE COEFFICIENTS}

\section{IIIA. Protons on Si, GaAs and InP}

NIEL calculations for protons in Si and comparisons with experimental data have been given previously[1] and will not be discussed further here. Anspaugh[23] has reported an extensive set of proton and electron relative damage 
coefficients for efficiency degradation in GaAs solar cells, which can be compared with the energy dependence of the NIEL, as shown for protons in figure 3 . The circles in figure 3 are data taken from figure 8 in reference [23] and the NIEL values have been normalized to these at $10 \mathrm{MeV}$. It can be seen that the experimental damage coefficients and the NIEL coincide closely from $100 \mathrm{MeV}$ down to $\sim 0.3 \mathrm{MeV}$, where the data first rise slightly above the calculation before dropping rapidly for $\mathrm{E}<0.05 \mathrm{MeV}$.

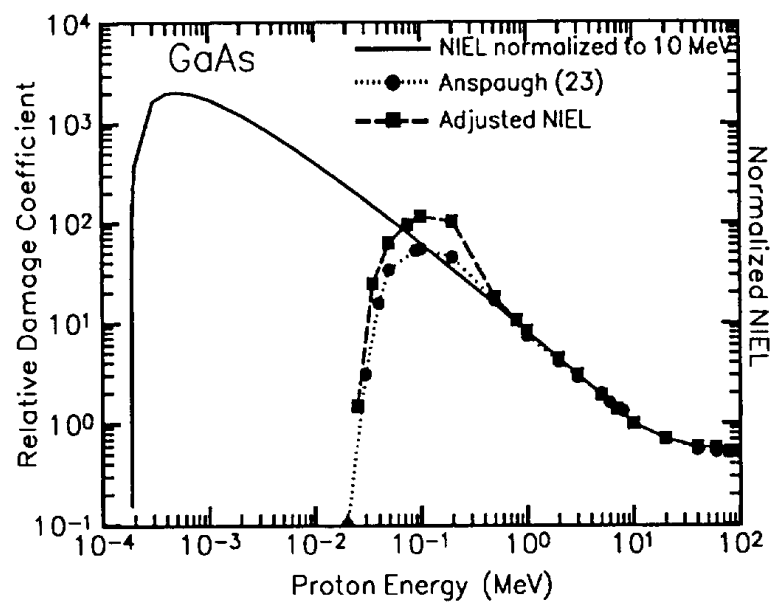

Figure 3 A comparison of the energy dependence of the calculated proton NIEL for GaAs with measured damage coefficients (circles) from Anspaugh[23]. The squares show an adjustment to the NIEL to account for the finite extent of the active volume of the device.

The rapid fall-off in damage coefficient occurs for unidirectional irradiation from the front, when the range of the incident protons is comparable to the depth of the active volume below the front surface of the cell. Lower energy protons will not be able to reach the active volume of the device and will therefore have no damaging effect. For the particular devices measured by Anspaugh, PC-1D modelling indicates that the active region extended from $\sim 0.28-1.48 \mu \mathrm{m}$ below the front surface. In order to account for protons that stop in this region, the total damage energy deposited in the active volume was calculated using TRIM for protons with energy in the range $0.025-0.25 \mathrm{MeV}$. The results are shown as the "adjusted NIEL calculation" (squares) in figure 3. It can be seen that the rapid fall-off with decreasing energy seen in the measurements is also apparent in the calculation, but that the slight rise in damage coefficient near $0.3 \mathrm{MeV}$ is somewhat overestimated. For approximate computational purposes, the simplest approach is to reduce the NIEL to zero for proton energies with a range less than the depth of the active volume.

The available data for InP devices is less extensive than for GaAs, but Yamaguchi's group has recently given some damage coefficients for InP diffused junction solar cells[22]. These data are compared to the energy dependence of the proton
NIEL in figure 4 . The similarity between figures 3 and 4 is clear, although there is more scatter in the InP data.

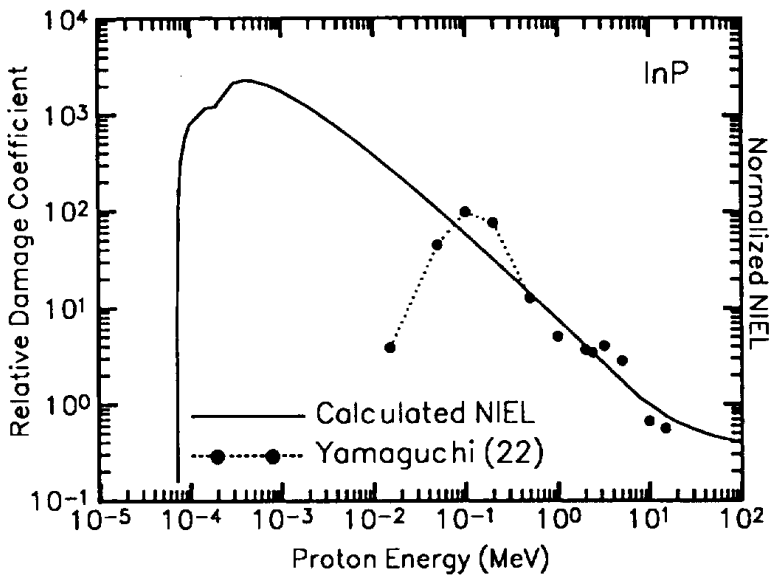

Figure 4 A comparison of the energy dependence of the calculated proton NIEL for InP with damage coefficients measured by Yamaguchi et al.[22]. The fall off in the data for energies $<10^{-1} \mathrm{MeV}$ is due to the finite extent of the active volume. (See figure 3 and the text).

The agreement between the experimental data and the calculations shown in figures 3 and 4 indicates that there is a linear dependence, i.e., direct proportionality, between the damage factors and the proton NIEL. The results in figure 3 and 4 are for efficiency degradation damage factors, but a similar linear dependence is also found for other parameters, such as the short circuit current, $\mathrm{J}_{\mathrm{sc}}$ and for other materials, such as $\mathrm{Si}$, as will be shown below. In all cases reported to date, the dataare consistent with a linear dependence of proton damage coefficient with NIEL, so that in principle only one measurement and the calculated NIEL are all that is required to determine the proton damage coefficients for all other energies. This result is very useful for predicting device degradation in complex space proton environments.

\section{IIIB. Electrons on GaAs and Si}

As figure 1 shows, the NIEL for electrons of a given energy is smaller by several orders of magnitude than the corresponding proton NIEL. The electron damage coefficients are therefore also expected to be much smaller than proton damage coefficients, and Anspaugh's data[23] confirm this conclusion. In figure 5 , the short circuit current damage coefficients for GaAs solar cells are plotted against the NIEL on a log-log plot, for both protons and electrons. The straight line on figure 5 was fitted to the proton data and extended to lower values. The slope of this line is near unity (1.08) as expected and it can be seen to pass through the electron data, although the electron data on their own appears to have a slope somewhat larger than unity. In general, however, the data in figure 5 tend to fall on a single line with a slope of unity, so that in principle a measurement made with one particle energy 
would enable the $\mathrm{J}_{\mathrm{sc}}$ damage coefficients for all other particle energies in GaAs to be calculated using the electron and proton NIEL.

Unlike the results for protons, however, a linear relationship between electron damage coefficients on NIEL is not always observed. $\mathrm{Si}$, in particular, shows an unusual behavior as can be seen in figure 6 . Figure 6 shows data from three separate reports. Asimov's[25] proton data and Carter and Downing's[26] electron data are all diffusion length damage coefficients in $1 \Omega . c m$ p-type Si. Figure 6 also shows Carter and Downing's data for n-type material. The proton data show the expected linear relationship with NIEL, but the electron data for p-type $\mathrm{Si}$, which was taken over the energy range from $0.6-40 \mathrm{MeV}$, show a quadratic dependence on the electron NIEL. This result is consistent with Carter's[27] observation that there was a second order dependence of the damage coefficient on the product of the cross section and the average number of secondary displacements for $n^{+} p$ Si solar cells. This product is analogous to NIEL.

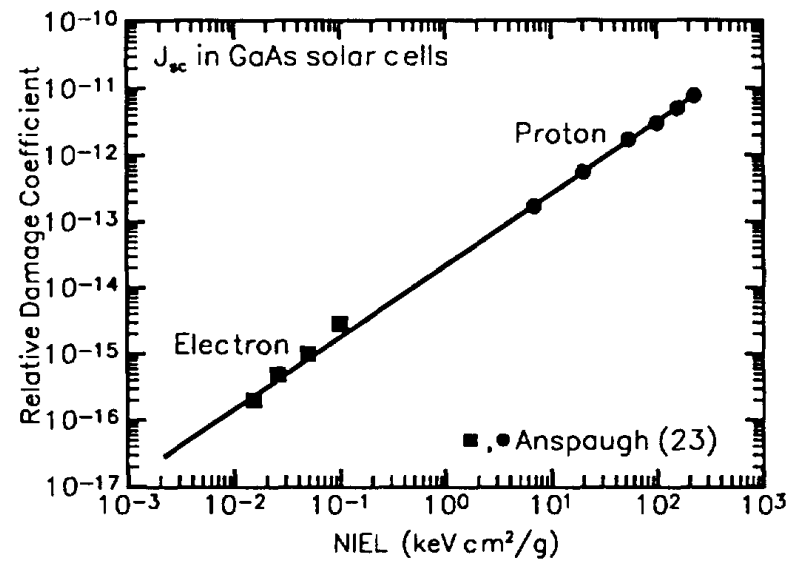

Figure 5 The linear relationship between the short circuit current damage coefficients and the NIEL for protons and electrons in GaAs solar cells[21].

Since Carter and Downing's data were taken nearly thirty years ago, it is possible that the results were due to the early manufacturing process used to produce the $\mathrm{Si}$. However, the behavior observed by Carter and Downing was confirmed by results recently obtained by Noguchi[26]. Noguchi measured the "critical fluence", $\phi_{\mathrm{xi}}$, for $\mathrm{J}_{\mathrm{sc}}$ for different energy electrons, i.e.,

$$
J_{s c}=J_{s c 0}\left\{1-C_{i} \ln \left(1+\phi / \phi_{x i}\right)\right\}
$$

where $C_{i}$ is a constant and $\phi$ is the electron fluence. It can be shown that $\phi_{x i}=1 / K_{L} L^{2}$, so the reciprocal of $\phi_{x i}$ is proportional to the diffusion length damage coefficient. Noguchi does not give information about the resistivity of the Si so it is necessary to normalize the data for comparison with Carter's and the normalization was made at $1 \mathrm{MeV}$. The results are shown in figure 6 , where the agreement with Carter and Downing's data is immediately apparent.

Both Carter's and Noguchi's measurements were made on p-type Si. N-type Si[26] shows qualitatively different behavior, however, as can be seen in figure 6 . It is clear that n-type Si shows an approximate linear dependence on NIEL also seen in proton irradiated material.

The data shown in figure 6 is representative of much more that have been reported in the literature for both junctions and bulk Si. Lugakov[29] gives lifetime damage coefficients for electrons over the range $2.5-1,200 \mathrm{MeV}$, which show a linear dependence on NIEL. Wyatt et al.[30] give both lifetime and carrier removal damage coefficients for electrons on $\mathrm{p}$ - and $\mathrm{n}$-type $\mathrm{Si}$, which are also consistent with the results shown in figure 6 .

\section{DISCUSSION}

The results presented here indicate that there is a direct proportionality between proton damage coefficients and the energy dependence of the NIEL for Si, GaAs and InP. This proportionality appears to hold for all parameters, e.g., solar cell efficiency, short circuit current, and bipolar transistor gain. To calculate device performance degradation in a proton environment, therefore, all that is required is the differential proton flux, a measurement made at one proton energy and the calculated NIEL.

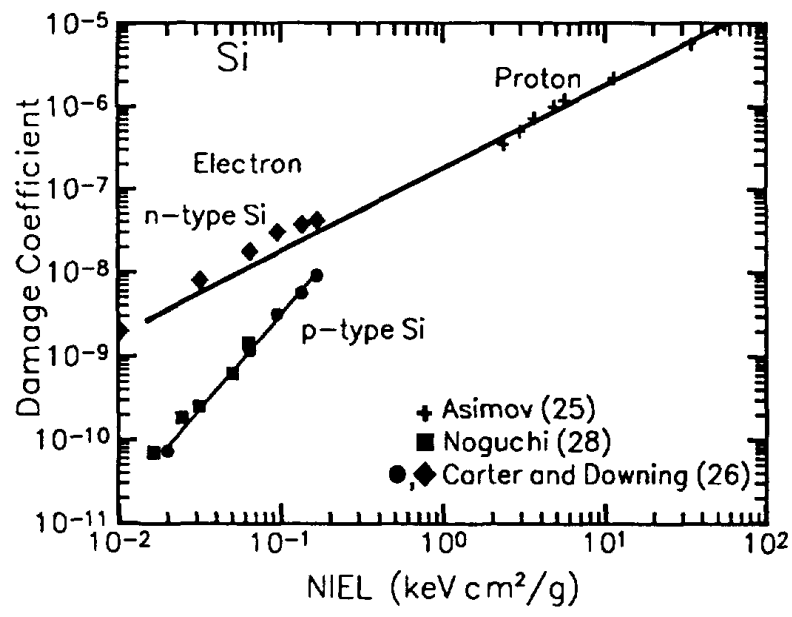

Figure 6 A plot showing the relationship between the NIEL and damage coefficients for $\mathrm{Si}$. The crosses[25] and the circles[26] are measured diffusion length damage coefficients for $1 \Omega . c m$ p-type Si. There is a linear relationship for protons and a quadratic relationship for electrons. The squares[28] are recent solar cell damage coefficients for p-type Si normalized for $1 \mathrm{MeV}$ electrons. The diamonds are diffusion length damage coefficients[24] for $1 \Omega$.cm n-type $\mathrm{Si}$ showing a linear relationship with NIEL.

There is less uniformity observed in electron damage results. For some parameters such as the short circuit current in $\mathrm{GaAs}$ or the lifetime damage coefficient in $\mathrm{n}$-type $\mathrm{Si}$, there 
is a linear relationship between damage coefficients and the energy dependence of the electron NIEL, and an extrapolation of the proton curve passes through the electron data. In general, however, this is not the case and in converting a 10 $\mathrm{MeV}$ equivalent proton fluence to a $1 \mathrm{MeV}$ electron equivalent fluence, as is often required for comparing solar cell performance, simply using the ratio of the $10 \mathrm{MeV}$ proton to $1 \mathrm{MeV}$ electron NIELs will usually overestimate the equivalent $1 \mathrm{MeV}$ electron fluence. For comparing electron and proton damage for a new technology, it is necessary, therefore, to make measurements at a minimum of two electron energies in order to determine both the functional dependence on NIEL and to find the quantitative relationship to the proton damage.

The reason for the different behavior observed in electron and proton damage appears to originate in the relatively much lower recoil energies produced by electrons. Wood et al.[31] have reported calculations using the Monte Carlo code MARLOWE, which indicate that recoils in $\mathrm{Si}$ with $\mathrm{E}_{\text {thresh }}<\mathrm{E}<$ $\sim 2 \mathrm{keV}$ produce only "free" point defects. Similarly, for recoils with $\sim 2<\mathrm{E}<\sim 12 \mathrm{keV}$, one defect cluster is formed and for recoils with $\mathrm{E}>-20 \mathrm{keV}$ a tree like defect structure is formed, with terminal clusters at the end of branch-like defect regions. For higher $\mathrm{Z}$ materials such as $\mathrm{GaAs}$, the comparable energies would be higher. These results suggest that if particle irradiation of $\mathrm{Si}$ produces mostly recoils with energies $<\sim 2$ $\mathrm{keV}$, the damage will be qualitatively different than for higher energy recoils. Such low energy recoils will generally dominate only for low energy ( $<\sim 5 \mathrm{MeV}$ ) electrons. When viewed in this way, it is not surprising that unusual behavior is observed in electron irradiations, in which the nature of the impurities present and the details of the crystal structure will strongly influence the type of defects produced.

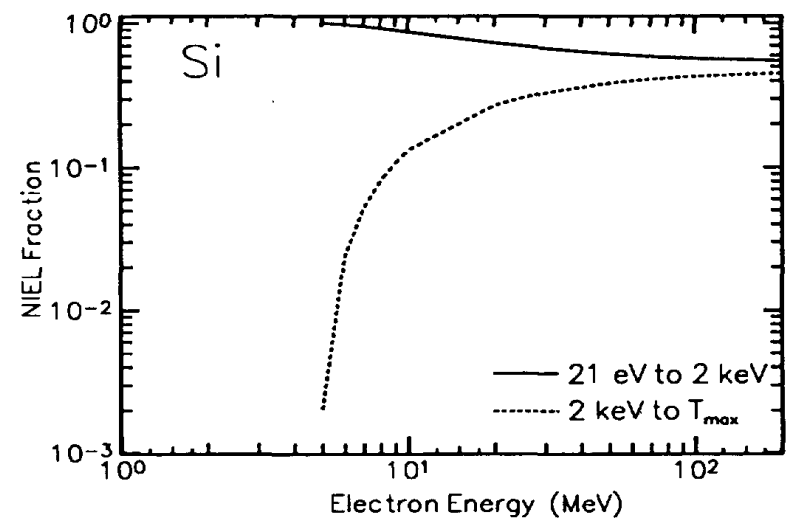

Figure 7 The fraction of the total NIEL contributed by recoils with energy from the threshold $(21 \mathrm{eV})$ to $2 \mathrm{keV}$, (continuous line), and from $2 \mathrm{keV}$ to the maximum, (broken line). The latter rises rapidly initially until $\mathrm{E} \sim 30 \mathrm{MeV}$, then levels out.

As has been noted in figure 6, the electron damage coefficients for $\mathrm{n}$ - and p-type Si show qualitatively different behavior. However, it can be seen that they approach each other at higher electron energies, i.e., as the energy of the recoils increases above $-2 \mathrm{keV}$. This result can be put on a more quantitative footing. The fraction of the NIEL produced in $\mathrm{Si}$ by recoils with energy $<2 \mathrm{keV}$ was calculated as a function of incident electron energy as shown in figure 7. Figure 7 shows, as expected, that for very low energy electrons, recoils with $\mathrm{E}<2 \mathrm{keV}$ cause nearly all the NIEL. However, as the electron energy rises from $10-40 \mathrm{MeV}$, the fraction of the NIEL coming from recoils with $\mathrm{E}>2 \mathrm{keV}$ increases rapidly, but levels off once the electron energy is -40 $\mathrm{MeV}$. This result indicates that once the incident electron energy is $>-40 \mathrm{MeV}$, the qualitative nature of the damage produced remains unchanged, as the work of Wood et al. would suggest. This result seems to consistent with all the available experimental data. Similar behavior would also be expected in other materials such as GaAs and InP.

The fact that there is a linear relationship between the electron damage coefficients and NIEL in n-type Si and an approximately quadratic dependence in p-type $\mathrm{Si}$, implies that different defects are responsible for the radiation response in each case. The linear dependence in n-type $\mathrm{Si}$ indicates the effect of a center whose introduction rate is directly proportional to the concentration of either interstitials or vacancies, such as the A-center (a substitutional oxygen atom$\mathrm{Si}$ vacancy pair). For p-type $\mathrm{Si}, \mathrm{Carter}[27]$ reported that the energy dependence of the damage coefficients in $n+p$ Si solar cells was similar to that of the $K$ center. Recent e.p.r. results indicate that the $K$ center consists of an interstitial oxygen atom bonded to an interstitial carbon atom [32]. The formation mechanism of such a center has not been fully elucidated, although the introduction rate by $1 \mathrm{MeV}$ electrons is only $-0.025 \mathrm{~cm}^{-1}$, indicating that the $\mathrm{K}$-center is not a primary defect. Also the second order dependence on NIEL suggests that the introduction rate is proportional to the product of the concentrations of both Si vacancies and interstitials. Since these concentrations should be proportional to each other, the introduction rate for the defect would be proportional to the square of the damage energy, i.e. the square of the NIEL. It should also be noted that the Si vacancy, Si interstitial and the $\mathrm{C}$ interstitial are all highly mobile at room temperature and that more than $90 \%$ of all vacancy-interstitial (Frenkel) pairs initially formed in $\mathrm{Si}$, recombine and do not lead to stable defects. Under these conditions, many formation mechanisms that could lead to a quadratic dependence on NIEL are possible. The resolution of this question awaits further work.

\section{SUMMARY}

Tabulated and graphical NIEL data are given in this paper which enable reliable displacement damage predictions to be made for $\mathrm{Si}, \mathrm{GaAs}$ and InP devices in any space radiation environment. For these semiconductors, analytic NIEL calculations for protons are extended down to the threshold energy for the first time. Previous calculations cut off at 1.0 $\mathrm{MeV}$. The analytic NIEL calculations have been compared with experimental data and with results from TRIM.

New electron NIEL calculations are provided for $\mathrm{Si}, \mathrm{GaAs}$ 
and InP, which both improve the accuracy of the results both near threshold and at higher energies where the losses to ionization are significant. Average NIEL values are provided for the first time for the electron spectrum generated in semiconductors by $\mathrm{Co}^{60}$ gamma radiation. This permits exposure in $\operatorname{rads}(\mathrm{Si})$ to be converted to an equivalent $1 \mathrm{MeV}$ electron fluence, thereby opening up a new experimental domain for exploring low damage rate effects.

\section{ACKNOWLEDGEMENTS}

This research was supported by the Office of Naval Rescanch.

\section{REFERENCES}

[1] G.P. Summers, E.A. Burke, C.J. Dale, E.A. Wolicki, P.W. Marshall and M.A. Gehlhausen, "Correlation of ParticleInduced Displacement Damage in Silicon," IEEE Trans. in Nucl. Sci., 34, 1134-1139 (1987).

[2] E.A. Burke, C.J. Dale, A.B. Campbell, G.P. Summers, T. Palmer and R. Zuleeg. "Energy Dependence of ProtonInduced Displacement Damage in GaAs" IEEE Trans. in Nucl. Sci., 34, 1220-1225 (1988).

[3] P.W. Marshall, C.J. Dale, G.P. Summers, E.A. Wolicki and E.A. Burke, "Proton, Neutron and ElectronInduced Displacement Damage in Germanium," IEEE Trans. in Nucl. Sci., 36, 1882-1888 (1989).

[4] R.J. Walters, S.R. Messenger, G.P. Summers, E.A. Burke and C.J. Keavney, "Space Radiation Effects in InP Solar Cells," IEEE Trans. in Nucl. Sci., 38, 1153-1158 (1991).

[5] G.P. Summers, E.A. Burke, D.B. Chrisey, M. Nastasi and J.R. Tesmer, "Effect of Particle-Induced Displacements on the Critical Temperature of $\mathrm{YBa}_{2} \mathrm{Cu}_{3} \mathrm{O}_{7}$," Appl. Phys. Letters, $55,1469-1471$ (1989).

[6] H.Y. Tada, J.R. Carter, B.E. Anspaugh and R.G. Downing, in The Solar Cell Radiation Handbook, JPL publication 82-69 (1982).

[7] G.P. Summers, E.A. Burke, M.A. Xapsos, C.J. Dale, P.W. Marshall, and E.L. Petersen, "Displacement Damage in GaAs Structures," IEEE Trans. in Nucl. Sci., 35, 1221-1226 (1988).

[8] F. Seitz and J.S. Koehler, in Solid State Physics, Vol. 2, edited by F. Seitz and D. Tumbull, (Academic Press, New York, 1956).

[9] J.W. Corbett and G.D. Watkins, "Production of Divacancies and Vacancies by Electron Irradiation of Silicon," Phys. Rev., 138A, 555-560 (1965).
[10] A.L. Barry, R. Maxseiner, R. Wojick, M.A. Briere and D. Braunig, "An Improved Displacement Damage Monitor," IEEE Trans. in Nucl. Sci., 37, 1726-1731 (1990).

[11] R. Bauerlein, "Displacement Thresholds in Semiconductors" in Radiation Damage in Solids, Edited by D.S. Billington, (Academic Press, New York 1962).

[12] J. Lindhard, V. Nielsen, M. Scarff and P.V. Tomsen, "Integral Equations Governing Radiation Effects, Notes on Atomic Collisions, III)," Mat. Fys. Medd. Dan. Vid. Selsk, $33, \mathrm{~N} 10,1-42$ (1963).

[13] N. F. Moth, "The Scattering of Fast Electrons by Atomic Nuclei", Proc. Roy Soc. Lond., A124, 425-442 (1929).

[14] W.A. Mckinley and H. Feshbach, "The Coulomb Scattering of Relativistic Electrons by Nuclei," Phys. Rev., 74, 1759-1763 (1948).

[15] R.M. Curr, "The Coulomb Scattering of High Energy Electrons and Positrons by Nuclei", Proc. Phys. Soc.( London), A68, 156-164 (1955).

[16] J.A. Doggett and L.V. Spencer, "Elastic Scattering of Electrons and Positrons by Point Nuclei", Phys. Rev., 103, 1597-1601 (1956).

[17] J.F. Ziegler, J.P. Biersack and U. Littmark, "The Stopping and Range of Ions in Solids, Volume 1", Pergammon Press, New York (1985).

[18] D.G. Doran, "Neutron Displacement Cross Sections for Stainless Steel and Tantalum Based on a Lindhard Model," Nucl. Sci. Eng., 49, 130-144 (1972).

[19] A.R. Sattler, F.L. Vook and J.M. Palms, "Ionization Produced by Energetic Germanium Atoms within a Germanium Lattice", Phys. Rev., 143, 588-594 (1966).

[20] J.F. Ziegler J.P. Biersack and U. Littmark, "TRIM-1990 (90.05)".

[21] Y. Yamaguchi and C. Amano, "Co-60 Gamma Ray and Electron Irradiation of GaAs Single Crystals and Solar Cells", J. Appl. Phys, 54, 5021-5029 (1983).

[22] M. Yamaguchi, C. Uemura and A. Yamamoto, "Radiation Damage in InP Single Crystals and Solar Cells", $J$. Appl. Phys., 55, 1429-1436 (1984)

[23] B.E. Anspaugh, "Proton and Electron Damage Coefficients for GaAs/Ge Solar Cells", Proceedings of Twenty-Second IEEE PVSC, 1593-1598 (1992).

[24] T. Takamoto et al., "Indium Phosphide Solar Cells for Space Applications", Technical Digest of the International 
PVSEC-5, Kyoto, Japan (1990); M. Yamaguchi et al., "Analysis of Space Flight Data of InP Solar CElls in EXOSD Orbit", Proceedings of Twenty-first IEEE PVSC, 15761581 (1991).

[25] K.S. Asimov, S.M. Gorodatskii, G.M. Grigor'eva, L.R. Kreinin and A.P. Landsman, "Influence of Disordered Regions on the Recombination in Proton-Irradiated p-type Silicon", Sov. Phys. Semicond., 7, 1021-1025 (1974).

[26] J.R. Carter and R.G. Downing, "Charged Particle Radiation Damage in Semiconductors, XI: Effect of Low Energy Protons and High Energy Electrons on Silicon", Interim Technical Final Report, TRW Space Technology Laboratories, May 1965.

[27] J.R. Carter, "Effect of Electron Energy on Defect Introduction In Silicon", J. Phys. Chem. Solids, 27, 913-918 (1966);
[28] T. Noguchi and M. Uesugi, "Electron Energy Dependence of Relative Damage Coefficients of Silicon Solar Cells for Space Use", Technical Digest of the International PVSEC.5, Kyoto, Japan (1990);

[29] P.F. Lugakov and I.M. Fillipov, "Radiation Defect Clusters in Electron-Irradiated Silicon", Radiation Effects, 90 , 297-305 (1985).

[30] M.E. Wyatt, V.A.J. van Lint and E .G. Walker, "Proton Correlation Studies", Techn. Rept. AFML-TR-66-77 (1966).

[31] S. Wood, N.J. Doyle, J.A. Spitznagel. W.J. Choyke, R.M. More, J.N. McGruer and R.B. Irwin, "Simulation of Radiation Damage in Solids," IEEE Trans. in Nucl. Sci., 28, 4107-4112 (1981).

[32] J.M. Trombetta and G.D. Watkins, "Identification of an Interstitial Carbon-Interstitial Oxygen Complex in Silicon," Appl. Phys. Letters, 51, 1103-1105 (1987).

\section{APPENDIX}

NIEL FOR PROTONS AND ELECTRONS IN Si, GaAs AND InP*

\begin{tabular}{|c|c|c|c|c|c|c|c|c|}
\hline \multirow[b]{2}{*}{ ENERGY(MeV) } & \multirow[b]{2}{*}{$\mathrm{Si}(21 \mathrm{eV})$} & \multicolumn{2}{|c|}{ PROTON (MeV.cm²/g) } & \multirow[b]{2}{*}{ InP } & \multirow[b]{2}{*}{$\mathrm{Si}(2 \mathrm{leV})$} & \multicolumn{3}{|c|}{ ELECTRON $\left(\mathrm{MeV} . \mathrm{cm}^{2} / \mathrm{g}\right)$} \\
\hline & & $\mathrm{Si}(12.9 \mathrm{eV})$ & GaAs & & & $\mathrm{Si}(12.9 \mathrm{eV})$ & GaAs & InP \\
\hline $1 \times 10^{-4}$ & - & 2.581 & - & 5.778 & - & - & - & - \\
\hline $1 \times 10^{-3}$ & $1.546 \times 10^{1}$ & $1.964 \times 10^{1}$ & $1.150 \times 10^{1}$ & $1.288 \times 10^{1}$ & - & - & - & - \\
\hline 2 & $1.053 \times 10^{1}$ & $1.262 \times 10^{1}$ & 8.071 & 8.757 & - & - & - & - \\
\hline 3 & 8.098 & 9.492 & 6.275 & 6.731 & - & - & - & - \\
\hline 5 & 5.659 & 6.496 & 4.434 & 4.706 & - & - & - & - \\
\hline 7 & 4.413 & 5.010 & 3.479 & 3.673 & - & - & - & - \\
\hline $1 \times 10^{-2}$ & 3.360 & 3.779 & 2.664 & 2.800 & - & - & - & - \\
\hline 2 & 1.938 & 2.147 & 1.551 & 1.619 & - & - & - & - \\
\hline 3 & 1.390 & 1.529 & 1.118 & 1.163 & - & - & - & - \\
\hline 5 & $9.058 \times 10^{-1}$ & $9.895 \times 10^{-1}$ & $7.338 \times 10^{-1}$ & $7.607 \times 10^{-1}$ & - & - & - & - \\
\hline 7 & $6.799 \times 10^{-1}$ & $7.396 \times 10^{-1}$ & $5.536 \times 10^{-1}$ & $5.729 \times 10^{-1}$ & - & - & - & - \\
\hline $1 \times 10^{-1}$ & $5.003 \times 10^{-1}$ & $5.421 \times 10^{-1}$ & $4.093 \times 10^{-1}$ & $4.228 \times 10^{-1}$ & - & - & - & - \\
\hline 2 & $2.722 \times 10^{-1}$ & $2.931 \times 10^{-1}$ & $2.251 \times 10^{-1}$ & $2.318 \times 10^{-1}$ & - & $9.741 \times 10^{-6}$ & - & $3.883 \times 10^{-6}$ \\
\hline 3 & $1.895 \times 10^{-1}$ & $2.034 \times 10^{-1}$ & $1.578 \times 10^{-1}$ & $1.622 \times 10^{-1}$ & $6.481 \times 10^{-6}$ & $1.850 \times 10^{-5}$ & $2.350 \times 10^{-6}$ & $6.713 \times 10^{-6}$ \\
\hline 5 & $1.194 \times 10^{-1}$ & $1.278 \times 10^{-1}$ & $1.005 \times 10^{-1}$ & $1.031 \times 10^{-1}$ & $1.629 \times 10^{-5}$ & $2.973 \times 10^{-5}$ & $1.177 \times 10^{-5}$ & $1.719 \times 10^{-5}$ \\
\hline 7 & $8.773 \times 10^{-2}$ & $9.371 \times 10^{-2}$ & $7.441 \times 10^{-2}$ & $7.624 \times 10^{-2}$ & $2.320 \times 10^{-5}$ & $3.453 \times 10^{-5}$ & $1.861 \times 10^{-5}$ & $2.482 \times 10^{-5}$ \\
\hline $1 \times 10^{0}$ & $6.381 \times 10^{-2}$ & $6.727 \times 10^{-2}$ & $5.402 \times 10^{-2}$ & $5.527 \times 10^{-2}$ & $3.142 \times 10^{-5}$ & $4.253 \times 10^{-5}$ & $2.656 \times 10^{-5}$ & $3.348 \times 10^{-5}$ \\
\hline 2 & $3.296 \times 10^{-2}$ & $3.506 \times 10^{-2}$ & $2.886 \times 10^{-2}$ & $2.944 \times 10^{-2}$ & $5.069 \times 10^{-5}$ & $6.137 \times 10^{-5}$ & $4.486 \times 10^{-5}$ & $5.223 \times 10^{-5}$ \\
\hline 3 & $2.243 \times 10^{-2}$ & $2.383 \times 10^{-2}$ & $1.993 \times 10^{-2}$ & $2.031 \times 10^{-2}$ & $6.366 \times 10^{-5}$ & $7.417 \times 10^{-5}$ & $5.655 \times 10^{-5}$ & $6.370 \times 10^{-5}$ \\
\hline 5 & $1.376 \times 10^{-2}$ & $1.460 \times 10^{-2}$ & $1.248 \times 10^{-2}$ & $1.269 \times 10^{-2}$ & $8.106 \times 10^{-5}$ & $9.147 \times 10-5$ & $7.182 \times 10^{-5}$ & $7.870 \times 10^{-5}$ \\
\hline 7 & $1.047 \times 10^{-2}$ & $1.107 \times 10^{-2}$ & $9.155 \times 10^{-3}$ & $9.374 \times 10^{-3}$ & $9.282 \times 10^{-5}$ & $1.031 \times 10^{-4}$ & $8.208 \times 10^{-5}$ & $8.879 \times 10^{-5}$ \\
\hline $1 \times 10^{1}$ & $7.885 \times 10^{-3}$ & $7.885 \times 10^{-3}$ & $6.588 \times 10^{-3}$ & $7.241 \times 10^{-3}$ & $1.051 \times 10^{-4}$ & $1.153 \times 19^{-4}$ & $9.301 \times 10^{-5}$ & $9.948 \times 10^{-5}$ \\
\hline 2 & $5.360 \times 10^{-3}$ & $5.360 \times 10^{-3}$ & $4.693 \times 10^{-3}$ & $4.682 \times 10^{-3}$ & $1.271 \times 10^{-4}$ & $1.373 \times 10^{-4}$ & $1.140 \times 10^{-4}$ & $1.199 \times 10^{-4}$ \\
\hline 3 & $4.778 \times 10^{-3}$ & $4.778 \times 10^{-3}$ & $4.028 \times 10^{-3}$ & $3.958 \times 10^{-3}$ & $1.381 \times 10^{-4}$ & $1.482 \times 10^{-4}$ & $1.257 \times 10^{-4}$ & $1.313 \times 10^{-4}$ \\
\hline 5 & $3.884 \times 10^{-3}$ & $3.884 \times 10^{-3}$ & $3.749 \times 10^{-3}$ & $3.356 \times 10^{-3}$ & $1.496 \times 10^{-4}$ & $1.597 \times 10^{-4}$ & $1.395 \times 10^{-4}$ & $1.448 \times 10^{-4}$ \\
\hline 7 & $3.161 \times 10^{-3}$ & $3.161 \times 10^{-3}$ & $3.640 \times 10^{-3}$ & $3.107 \times 10^{-3}$ & $1.555 \times 10^{-4}$ & $1.657 \times 10^{-4}$ & $1.476 \times 10^{-4}$ & $1.529 \times 10^{-4}$ \\
\hline $1 \times 10^{2}$ & $2.598 \times 10^{-3}$ & $2.598 \times 10^{-3}$ & $3.490 \times 19-3$ & $3.005 \times 10^{-3}$ & $1.603 \times 10^{-4}$ & $1.704 \times 10^{-4}$ & $1.550 \times 10^{-4}$ & $1.606 \times 10^{-4}$ \\
\hline 2 & $1.940 \times 10^{-3}$ & $1.940 \times 10^{-3}$ & $3.930 \times 10^{-3}$ & $3.537 \times 10^{-3}$ & $1.651 \times 10^{-4}$ & $1.751 \times 10^{-4}$ & $1.660 \times 10^{-4}$ & $1.721 \times 10^{-4}$ \\
\hline
\end{tabular}

